\title{
Diffuse Iris Melanoma: Conservative Treatment with Proton Beam Therapy after Limbal Stem Cell Preservation or Enucleation?
}

\author{
Alice Leblanc a, c Livia Lumbroso-Le Rouic ${ }^{a} \quad$ Laurence Desjardins $^{a}$ \\ Rémi Dendale $^{b}$ Nathalie Cassoux ${ }^{a, d}$ \\ ${ }^{a}$ Département d'Ophtalmologie, Institut Curie, Paris, France; ${ }^{b}$ ICPO - Centre de Protonthérapie de I'Institut Curie à \\ Orsay, Paris, France; ' Département d'Ophtalmologie, Institut Curie, Poitiers, France; ${ }^{\mathrm{d}}$ Université Paris V Descartes, \\ Paris, France
}

\section{Keywords \\ Diffuse iris melanoma - Proton beam therapy · \\ Limbal stem cell deficiency}

\begin{abstract}
Objectives: Iris melanomas represent 2-3\% of uveal melanomas; the diffuse variant accounts for approximately $10 \%$ of all iris melanomas. Different treatment modalities for diffuse iris melanomas (DIM) have been proposed depending on the local status as well as the age and general condition of the patient. Methods: This study is a single-centre retrospective case series describing the diagnosis, treatments and outcomes of DIM. Treatment consisted of enucleation or proton beam therapy (PT) of the whole anterior segment. Patients who were treated with PT benefitted from limbal stem cell preservation before irradiation. Results: Between 1996 and 2016, a total of 14 patients with DIM presented to our institution and were included in the database. The global survival was $86 \%$. The median follow-up was 4.6 years (range 4 months to 15 years). Only 1 patient (7\%) developed metastatic disease of the DIM (gastric location). No patient developed liver metastasis. Seven patients were treated by enucleation and 7 by PT after limbal stem cell preservation. After
\end{abstract}

\section{KARGER}

(c) 2019 S. Karger AG, Basel

E-Mail karger@karger.com

www.karger.com/oop a conservative attempt, local tumour recurrence occurred in 2 patients at 2 years, requiring enucleation. The cornea was clear after irradiation in all patients. Cataract $(n=6)$ and glaucoma $(n=4)$ were the main complications after irradiation. Conclusions: DIM is a very rare tumour. The global survival is excellent. Conservative treatment with PT is an efficient alternative to enucleation and allows good local tumour control. Cataract and glaucoma are the main radiation-related complications, but the corneal status was excellent due to the stem cell harvest prior to radiotherapy.

ㄷ) 2019 S. Karger AG, Basel

\section{Introduction}

Iris melanomas represent $2-3 \%$ [1] of uveal melanomas; the diffuse variant accounts for approximately $10 \%$ of all iris melanomas. The definition of diffuse iris melanoma (DIM) varies according to the different published articles. For the majority of the authors, DIM consists of a flat, infiltrating growing iris lesion with seeding throughout the anterior chamber [2]. These patients may show progressive heterochromia and unilateral glaucoma secondary to angle invasion [3]. Other 
authors also include patients with a measurable iris lesion involving more than 5 clock hours or associated to anterior chamber spread.

Different treatment modalities for DIM have been proposed depending on the local status of the affected eye and its fellow eye as well as the age and general condition of the patient. Radiation therapy either by proton beam therapy (PT) [4] or brachytherapy [5] can be used as conservative treatment in order to preserve the eyeball and vision. Whole anterior segment irradiation by PT can be a useful alternative to enucleation for DIM in terms of eye preservation with good local tumour control rates, but high rates of glaucoma, cataract and varying degrees of limbal stem cell failure were described by Konstantinidis et al. [4].

The aim of this study was to describe the management and outcomes of a series of patients with DIM in 1 single tertiary referral centre treated with a conservative attempt by PT or by enucleation.

\section{Materials and Methods}

This study is a single-centre retrospective case series describing the diagnosis, treatments and outcomes of DIM. All patients presenting with iris melanoma were prospectively registered in a database. At diagnosis, all patients signed an informed consent for the use of their clinical and biologic data for research and publication. The database was declared to the CNIL according to the French legislation. For this study, only DIM patients were selected, and data were extracted from the database. Patients with a previously treated lesion or lost to follow-up were not included in this study. Patients with ciliary body or choroidal melanoma with an iris extension were excluded from this study.

DIM was defined as patients presenting either with a diffuse insidious hyperchromic lesion of the entire iris or with multiple foci of iris lesions or with a measurable lesion associated to the invasion of the iridocorneal angle on $360^{\circ}$. Complete ocular examination was performed at diagnosis, including visual acuity, intraocular pressure (IOP) measurement, slit-lamp examination (tumour localization, tumour colour, iris colour) and gonioscopy in order to determine the extent of the angle invasion. A colour photography of the anterior segment and a high-frequency B-scan ultrasonography were performed.

Treatment consisted of enucleation in the first years of the study and of either enucleation or conservative treatment with PT of the whole anterior segment afterwards (from 2012 until the end of the study). Patients who were treated with PT benefitted from limbal stem cell harvest before irradiation.

Data collected at diagnosis included:

- demographic features: age, gender and presence of an iris nevus in the medical history;

- the baseline ocular exam, including visual acuity, IOP (and need for medical treatment or not), iris colour, presence of heterochromia, tumour localization, tumour colour, presence of a cataract, the extent of iridocorneal angle invasion by pigmented seeding (degrees) and the presence of extra-scleral extension;

Diffuse Iris Melanoma Treatment
- the high-frequency B-scan ultrasonography results (thickness of the lesion, the extent to the ciliary body);

- an iris biopsy, which was performed whenever it was needed to confirm the diagnosis.

Patients treated with a conservative approach by PT underwent surgery for limbal cell harvest before irradiation. Five days before PT, surgery under general anaesthesia was performed in order to remove 2 corneal stem cell samples at 12 and 6 o'clock. The grafts were stored in culture media (corneal chamber ${ }^{\circledR}$, Alchimia Srl, Italy).

PT was then realized after 3-dimensional modelling of the eyeball volume and the tumour volume based on the preliminary imaging examinations performed at the initial visit (photography, ultrasonography). The treated volume was the whole anterior segment surrounded by a $2.5-\mathrm{mm}$ safety margin. The dose distribution was based on a 3-dimensional reconstruction of the eye using EYEPLAN software. The doses delivered to the main intra-ocular structures (lens, ciliary body, cornea, optic disk and macula) were recorded. The irradiation technique does not need tantalum clip on sclera. Patients were treated with a standard protocol of $60 \mathrm{~Gy}$ external beam radiation therapy in 4 fractions of 15 Gy on 4 consecutive days. The patient fixed a luminous infrared diode with 1 eye. Irradiation was performed without pupillary dilatation. Irradiation was performed in the sitting position with topical anaesthesia (oxybuprocaine eye drops) allowing insertion of eyelid retractors in order to exclude the eyelids from the radiation fields. On the last day of PT, the limbal grafts were replaced after the last irradiation session.

Enucleation was realized under general anaesthesia. It consisted of a classic surgery with orbital implantation. Data collected included the results of the histopathologic examination (melanoma cell type: epithelioid, spindle or mixed), the presence or not of extra-scleral extension (and the need for complementary orbital radiotherapy).

Follow-up included an ophthalmological examination every 6 months in the first 2-3 years and then annually as well as general controls (liver B-scans were repeated every 6 months).

Data collected included survival and the appearance of metastasis in all patients. For the patients treated conservatively, data collected also included a general ocular examination: local tumour status, visual acuity, IOP, the need for glaucoma treatment (medical/ surgical) and possible ocular complications, such as loss of eyelashes, corneal status, dry eye, neovascular glaucoma, cataract, retinal detachment, radiation papillopathy and radiation retinopathy.

\section{Results}

Between 1996 and 2016, a total of 14 patients with DIM presented to our institution and were included in the database. The median follow-up was 4.6 years (range 4 months to 15 years). Seven patients had a conservative treatment with PT and limbal stem cell preservation. Seven patients were enucleated. General characteristics at baseline are summarized in Table 1. There were no bilateral cases.

Eight patients presented with a documented growth of an old and known iris lesion. All patients presented with iris heterochromia, with the affected eye darker than the 
Table 1. Demographics, general characteristics and tumour characteristics at diagnosis; VA and IOP at diagnosis and last follow-up

\begin{tabular}{|c|c|c|}
\hline & $\begin{array}{l}\text { Enucleation } \\
(n=7)\end{array}$ & $\begin{array}{l}\mathrm{PT} \\
(n=7)\end{array}$ \\
\hline Mean age, years & 68 & 60 \\
\hline \multicolumn{3}{|l|}{ Gender of the patients } \\
\hline Female & 1 & 1 \\
\hline Male & 6 & 6 \\
\hline \multicolumn{3}{|l|}{ Iris colour } \\
\hline Blue & 5 & 5 \\
\hline Green & 1 & 1 \\
\hline Brown & 0 & 1 \\
\hline Unknown & 1 & 0 \\
\hline \multicolumn{3}{|l|}{ Localization of the tumour } \\
\hline Right eye & 4 & 3 \\
\hline Left eye & 3 & 4 \\
\hline \multicolumn{3}{|l|}{ Follow-up data, years } \\
\hline Mean (range) & $6.1(0.33-15)$ & $2.4(1.5-3)$ \\
\hline \multicolumn{3}{|l|}{ Tumour type } \\
\hline Diffuse & 6 & 6 \\
\hline Multifocal & 1 & 1 \\
\hline Achromic component & 2 & 1 \\
\hline \multicolumn{3}{|c|}{ Extent of angle invasion by tumour seeding } \\
\hline $360^{\circ}$ & 3 & 5 \\
\hline $270^{\circ}$ & 1 & 1 \\
\hline $180^{\circ}$ & 1 & 1 \\
\hline $90^{\circ}$ & 1 & 0 \\
\hline $0^{\circ}$ & 0 & 0 \\
\hline Mean tumour thickness, mm & 2 & $1.02(0.54-1.3)$ \\
\hline \multicolumn{3}{|l|}{$\mathrm{VA}$, at diagnosis/final } \\
\hline $20 / 20$ to $20 / 40$ & $4 /-$ & $5 / 0$ \\
\hline $20 / 50$ to $20 / 100$ & $3 /-$ & $1 / 2$ \\
\hline $20 / 200$ or worse & $0 /-$ & $0 / 5$ \\
\hline Unavailable & $0 /-$ & $1 / 0$ \\
\hline \multicolumn{3}{|l|}{ IOP } \\
\hline$\leq 20 \mathrm{~mm} \mathrm{Hg}$ & $1 /-$ & $3 / 3$ \\
\hline $21-30 \mathrm{~mm} \mathrm{Hg}$ & $2 /-$ & $3 / 1$ \\
\hline $30-35 \mathrm{~mm} \mathrm{Hg}$ & $2 /-$ & $1 / 3$ \\
\hline$\geq 35 \mathrm{~mm} \mathrm{Hg}$ & $2 /-$ & $0 / 0$ \\
\hline
\end{tabular}

VA, visual acuity; IOP, intra-ocular pressure; PT, proton beam therapy.

fellow eye. All lesions were pigmented, but an achromic component was present in 3 of them. Pigment seeding in the iridocorneal angle at a distance from the tumour was observed in 13 patients. Tumour characteristics are summarized in Table 1.

At diagnosis, no patient presented with cataract. Visual acuity and IOP measurements are detailed for the 2 groups in Table 1. Medical treatment was necessary for 8 patients to reduce the IOP. Two patients underwent iris biopsy. The pathological exam reported melanoma with epithelioid cells in both cases.

All patients had high-frequency B-scans. In 4 patients, the lesion was in contact with the ciliary body but did not invade it. One patient presented a ciliary body invasion as well. For these patients treated by PT, the ciliary body was also included in the radiation field. At the end of followup, 1 patient developed metastasis from the iris melanoma (gastric location). No patient developed liver metas- 
Table 2. Results after proton beam therapy

\begin{tabular}{ll}
\hline Total of patients & 7 \\
Local recurrence & $2 / 7$ \\
Eye retention & $5 / 7$ \\
Local complication & \\
$\quad$ Cornea & $0 / 7$ \\
$\quad$ Cataract & $6 / 7$ \\
$\quad$ Glaucoma & $4 / 7$ \\
\hline
\end{tabular}

tasis from the iris melanoma. Two of the 14 patients developed another malignant tumour, one of them with associated metastasis. Two patients died during followup, one of gastric metastasis from melanoma, and for the second patient the reason is unknown.

The tumour was managed by conservative treatment in 7 cases. Median follow-up was 2.5 years (range 1.5-3 years). One patient developed a metastasis from DIM (gastric location). This patient died from metastatic disease during the follow-up. No patient developed liver metastasis from the DIM, and 1 patient developed a sigmoid colon adenocarcinoma with liver metastasis. Local tumour control was achieved in 5 patients. Local tumour recurrence occurred in 2 patients after 2 years of followup, requiring enucleation. The recurrence was observed in the ciliary body and the choroid. One of these 2 patients clinically presented with intra-ocular haemorrhage after cataract and glaucoma surgery. This patient required secondary enucleation because of ocular pain due to high IOP. Pathology showed active tumour with scleral exteriorization requiring orbital irradiation after surgery.

Mean visual acuity was 20/100 two years after PT mainly due to cataract. Cataract appeared in 6 patients, and 2 patients required surgery. Final visual acuity and IOP are summarized in Table 1 . Three patients with high IOP were treated by medical treatment and 1 with medical treatment and surgery (Molteno implant).

Two patients lost some eyelashes. The cornea was clear after treatment in all patients without any limbal stem cell deficiency (Table 2). No patient required treatment for dry eye or uveitis. No radiation papillopathy or radiation retinopathy occurred.

The tumour was managed by enucleation in 7 cases. Median follow-up after treatment was 4 years (range 0.33-15 years). Pathological exam reported melanoma with epithelioid cells in 2 patients, spindle-shaped cells in 4 patients and mixed cells in 1 patient. Extra-scleral extension was present in 1 patient, needing orbital irradia-

Diffuse Iris Melanoma Treatment
Table 3. Results at the end of follow-up ( 4.6 years)

$\begin{array}{lc}\text { Total of patients } & 14 \\ \text { Global survival } & 86 \% \\ \text { Melanoma-related deaths } & 1 / 14 \\ \text { Metastasis } & 1 / 14 \\ \quad \text { Liver metastasis of the DIM } & 0 / 14 \\ \quad \text { Gastric metastasis of the DIM } & 1 / 14\end{array}$

DIM, diffuse iris melanoma.

tion by photons. No local orbital complication was observed during follow-up. No patient developed metastasis from the DIM, but 1 patient developed Lieberkühn adenocarcinoma with liver metastasis. One patient died; the cause of death could not be obtained (Table 3 ).

\section{Discussion}

Iris melanoma is the most common malignant tumour of the iris [6]. It is a rare tumour representing only $2-3 \%$ of all uveal melanomas [5]. The diffuse variant is even more rare as it accounts for approximately $10 \%$ of all iris melanomas [5]. At our centre, 14 patients were treated for a DIM. The main strength of this study was the relatively large number of patients treated for such a rare disease either by enucleation or conservative treatment with PT and stem cell preservation. The retrospective design of this study is of course a shortcoming, but the results especially on the local ocular tolerance after PT are very interesting.

The 10-year survival probability of iris melanoma (focal and diffuse) was about 95\% [7]. In our study, the global survival was $86 \%$ with a median follow-up of 4.6 years, which confirmed that iris melanoma, even in its diffuse variant, has a better survival than choroidal melanoma (52-68\%) [8].

In our study, only 1 patient (7\%) developed metastatic disease from the DIM (gastric location), but none to the liver. Metastatic disease in iris melanoma is rare and is more frequently observed in DIM; the incidence of metastasis in DIM has been reported to be approximately $13 \%$ at 6 years [2].

Our series also emphasizes the low risk of metastatic disease in iris melanoma even in its diffuse variant, but a longer follow-up is needed as our median follow-up is 4.6 years (range 0.33-15 years). Two patients developed a second primary cancer (1 with liver metastasis). No pa- 
tient developed liver metastasis from the iris melanoma. Two patients died during follow-up, one from gastric metastasis from melanoma, and for the second patient the reason is unknown.

Treatment options for DIM have been proposed depending on several parameters, such as the status of the affected eye and its fellow eye as well as the age and general condition of the patient and the period of the study. Enucleation was the only option for several years; conservative therapies appeared later either with plaque radiotherapy or PT [7].

In our series, 5 patients were treated by enucleation before the development of conservative treatment. Later in the study period, some patients could be treated conservatively with PT and stem cell harvest (in order to decrease the possible anterior segment irradiation complications) as an alternative to enucleation.

PT is more appropriate than plaque brachytherapy because the radiation dose delivered to the tumour volume is homogeneous. With brachytherapy, the dose delivered at the base of the irradiated tumour is much higher than the needed dose at the apex; therefore, brachytherapy induces an overdose at the base of the lesion.

Published data on conservative treatment for DIM are scarce, as it is such a rare disease. A few case reports of conservative treatment have been reported either with plaque therapy or PT [5]. In this subset of treated patients, local tumour control was obtained in 5 patients in our series. Only 2 patients developed a local tumour recurrence in the ciliary body, needing enucleation. In patients treated by enucleation, no local orbital complication was observed during follow-up.

After conservative treatment by plaque radiotherapy, Shields et al. [7] reported that enucleation was necessary in $13 \%$ of patients at 5 years due to tumour recurrence $(n=3)$ and patient preference $(n=1)$ in 38 patients $(14$ of 38 patients treated had the diffuse variant). The treated volume was the whole anterior segment.

A study from Rundle et al. [9] reported that the local control rate for non-resectable iris melanomas (4 focal and 11 diffuse) was $93 \%$, and retention of the eye was $80 \%$ after PT. The treated volume was also the whole anterior segment.

The main complications of conservative treatment in our series were cataract ( 6 patients) and glaucoma (4 patients). Two patients needed cataract surgery. The IOP was not satisfactorily controlled with medical treatment in 3 patients (IOP between 23 and $30 \mathrm{~mm} \mathrm{Hg}$ ), and surgery was necessary in 1 patient (Molteno implant). None of the patients presented with neovascular glaucoma.
High IOP is a major concern in the management of iris melanomas. Rundle et al. [9] described PT of 15 cases defined as "non-resectable" iris melanomas. Eleven of 15 cases were classified as diffuse. Secondary glaucoma was the most common complication (53\%).

A study from Shields et al. [5] shows that up to $60 \%$ of cases with raised IOP require enucleation. The same centre reported, in another study, patients with "non-resectable" iris melanomas: $37 \%$ were diffuse and treated with a custom-designed iodine- 125 plaque [7]. At 5 years, radiation-related complications included neovascular glaucoma in $8 \%$. The aetiology of elevated IOP after treatment appears to be a combination of both tumour seeding into the angle and radiation effects on the angle structure.

Konstantinidis et al. [4] reported that glaucoma required treatment in 11 of 12 patients. In 10 patients, the IOP was controlled with eye drops, and 1 patient required surgical intervention with a Baerveldt implant. Cataract represents the second radiation-related complication. Cataract appeared in 6 of 7 patients, and 2 patients required surgery. Shields et al. [7] reported that radiationrelated complications included cataract in $70 \%$ of patients after conservative treatment with plaque radiotherapy. Rundle et al. [9] reported that cataract represented $20 \%$ of radiation-related complications.

The PT included irradiation of almost the entire ciliary body, but it did not lead to hypotonia in any case. The radiation field was increased to include the ciliary body if the iris lesions were in contact or invaded. No differences in terms of local complications were observed. No patients had uveitis, dry eye or neovascular glaucoma. The cornea was clear in all our patients with no dry eye syndrome or keratitis after PT and limbal cell stem preservation despite the fact that the limbus received the full irradiation dose. Willerding et al. [10], in their study of total anterior segment irradiation with PT but without stem cell harvest, reported epitheliopathy with signs of limbal stem cell insufficiency occurring in 1 patient and transient corneal erosion in 2 patients (out of 54 patients). Dry eye occurred in all their patients. Corneoscleral necrosis, radiation retinopathy, papillopathy or hypotony were not observed. Their mean follow-up was 4.6 years as in our series. Rundle et al. [9] described dry eyes in $27 \%$ of patients treated by PT.

Sandinha et al. [11] discuss the fact that higher doses of radiation would eliminate local recurrence but would increase the incidence of limbal stem cell deficiency and other complications. Konstantinidis et al. [4] reported 4 patients developing limbal stem cell deficiency after whole anterior segment PT for DIM in 12 patients. Singh 
et al. [12] reported the outcome of a patient treated by PT after limbal stem preservation for DIM. The cornea was clear after treatment. Shields et al. [7] reported corneal opacity in 3 of 38 patients (8\%). Petousis et al. [13] reported a case of multifocal iris melanoma treated by total anterior segment palladium-103 plaque irradiation therapy without significant complications on the cornea.

In our experience, the limbal stem cell preservation before PT allowed to avoid local major corneal complications as no patient had clinical signs of limbal stem cell deficiency or dry eye. The absence of radiation retinopathy and papillopathy was in accordance with the calculated irradiation doses (none received by these structures).

\section{Conclusion}

DIM is a very rare tumour, the global survival is good, and the eye can be treated conservatively with PT, which is an efficient alternative to enucleation. It allows good local tumour control with few complications. Cataract and glaucoma were the main radiation-related complica- tions, but the corneal status was excellent thanks to the stem cell harvesting realised prior to radiotherapy. A longer follow-up is needed in order to confirm the excellent results in terms of long-term local control and corneal tolerance as well as the evolution of IOP.

\section{Statement of Ethics}

All patients referred to our oncology center with the diagnosis of iris melanoma were prospectively enrolled in the Institut Curie database: MACRO (version 3.0.75) Infermed ${ }^{\circledR}$. Each patient gives his consent, at the diagnosis, for the use of the clinical data. Moreover, the study patients have been fully informed of possible studies, and gave their consent in participating. The approval of the ethics committee has been obtained.

The described research methods and analysis adhered to the tenets of the Declaration of Helsinki.

\section{Disclosure Statement}

None of the authors have any conflicts of interests in the matters discussed in the paper.

\section{References}

1 Shields CL, Materin MA, Shields JA, Gershenbaum E, Singh AD, Smith A. Factors associated with elevated intraocular pressure in eyes with iris melanoma. Br J Ophthalmol. 2001 Jun; 85(6):666-9.

2 Demirci H, Shields CL, Shields JA, Eagle RC Jr, Honavar SG. Diffuse iris melanoma: a report of 25 cases. Ophthalmology. 2002 Aug; 109(8):1553-60.

3 Skalicky SE, Giblin M, Conway RM. Diffuse iris melanoma: report of a case with review of the literature. Clin Ophthalmol. 2007 Sep; 1(3):339-42.

4 Konstantinidis L, Roberts D, Errington RD, Kacperek A, Damato B. Whole anterior segment proton beam radiotherapy for diffuse iris melanoma. Br J Ophthalmol. 2013 Apr; 97(4):471-4.
5 Shields CL, Shields JA, Materin M, Gershenbaum E, Singh AD, Smith A. Iris melanoma: risk factors for metastasis in 169 consecutive patients. Ophthalmology. 2001 Jan;108(1): 172-8.

6 Duke JR, Dunn SN. Primary tumors of the iris. AMA Arch Opthalmol. 1958 Feb;59(2): 204-14.

7 Shields CL, Naseripour M, Shields JA, Freire J, Cater J. Custom-designed plaque radiotherapy for nonresectable iris melanoma in $38 \mathrm{pa}-$ tients: tumor control and ocular complications. Am J Ophthalmol. 2003 May;135(5): $648-56$.

8 Bensoussan E, Thariat J, Maschi C, Delas J, Schouver ED, Hérault J, et al. Outcomes After Proton Beam Therapy for Large Choroidal Melanomas in 492 Patients. Am J Ophthalmol. 2016 May;165:78-87.

9 Rundle P, Singh AD, Rennie I. Proton beam therapy for iris melanoma: a review of 15 cases. Eye (Lond). 2007 Jan;21(1):79-82.
10 Willerding GD, Cordini D, Hackl C, Karle B, Lakotka N, Foerster MH, et al. Proton beam radiotherapy of diffuse iris melanoma in $54 \mathrm{pa}$ tients. Br J Ophthalmol. 2015 Jun;99(6):812-6.

11 Sandinha MT, Kacperek A, Errington RD, Coupland SE, Damato B. Recurrence of iris melanoma after proton beam therapy. $\mathrm{Br} \mathrm{J}$ Ophthalmol. 2014 Apr;98(4):484-7.

12 Singh AD, Dupps WJ Jr, Biscotti CV, Suh JH, Lathrop KL, Nairn JP, et al. Limbal Stem Cell Preservation During Proton Beam Irradiation for Diffuse Iris Melanoma. Cornea. 2017 Jan; 36(1):119-22.

13 Petousis V, Finger PT, Milman T. Multifocal iris melanoma treated with total anterior segment palladium-103 plaque radiation therapy. Graefes Arch Clin Exp Ophthalmol. 2011 Jun;249(6):937-40. 